\title{
CPAP and measures of cardiovascular risk in males with OSAS
}

\author{
M. Kohler*, , J.C.T. Pepperell ${ }^{\#,+}$, B. Casadei ${ }^{\mp}$, S. Craig*, N. Crosthwaite*, \\ J.R. Stradling*, and R.J.O. Davies*,\$
}

ABSTRACT: Obstructive sleep apnoea syndrome (OSAS) has been associated with hypertension, stroke and myocardial ischaemia in epidemiological and observational studies. Continuous positive airway pressure (CPAP) is the treatment of choice for OSAS, but the impact of this intervention on established risk factors for cardiovascular disease remains incompletely understood.

A total of 102 males with moderate-to-severe OSAS were randomised to therapeutic $(n=51)$ or subtherapeutic $(n=51)$ CPAP treatment for 4 weeks to investigate the effects of active treatment on 24-h urinary catecholamine excretion, baroreflex sensitivity (BRS), arterial stiffness (augmentation index) and 24-h ambulatory blood pressure (ABP).

After 4 weeks of therapeutic CPAP, significant reductions were seen in urine normetanephrine excretion (from mean \pm SD $179.7 \pm 80.1$ to $132.7 \pm 46.5 \mu \mathrm{mol} \cdot \mathrm{mol}^{-1} \mathrm{creatinine}$ ) and augmentation index (from $14.5 \pm 11.3$ to $9.1 \pm 13.8 \%$ ) compared with the subtherapeutic control group. Furthermore, therapeutic CPAP significantly improved BRS (from $7.1 \pm 3.3$ to $8.8 \pm 4.2 \mathrm{~ms} \cdot \mathrm{mmHg}^{-1}$ ) and reduced mean arterial ABP by $2.6 \pm 5.4 \mathrm{mmHg}$.

In conclusion, treatment of obstructive sleep apnoea with continuous positive airway pressure may lower cardiovascular risk by reducing sympathetic nerve activity, ambulatory blood pressure and arterial stiffness and by increasing sensitivity of the arterial baroreflex.

KEYWORDS: Arterial stiffness, baroreflex, catecholamines, continuous positive airway pressure, obstructive sleep apnoea

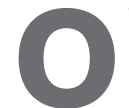

bstructive sleep apnoea syndrome (OSAS) is characterised by repetitive apnoea/hypopnoea during sleep associated with oxygen desaturations and sleep disruption. It has been estimated that $2-4 \%$ of the adult population in Western countries suffer from clinically significant OSAS, and it is becoming more prevalent as the average bodyweight of the population rises [1].

OSAS has been associated with hypertension, stroke and myocardial ischaemia in epidemiological and prospective observational studies [1, 2]. The pathophysiological mechanisms underlying the association between OSAS and cardiovascular disease are not fully understood, and indeed there may not be a causal relationship. During the actual repetitive episodes of apnoeas there is increased inspiratory effort, episodic hypoxaemia, recurrent arousals, reflex sympathetic activation, increased arterial stiffness and consequent marked transient increases in arterial blood pressure [3-5]. The prolonged repetitive rises in blood pressure are likely to induce excessive vascular shear stress, which has been shown to contribute to the formation of atherosclerotic plaques [6].

Sympathetic activity has been shown to be increased even in the daytime in patients with OSAS, both from measurements of circulating catecholamines and sympathetic nerve traffic. Augmented sympathetic activation may increase arterial stiffness and blunt baroreflex sensitivity (BRS), both of which may contribute to the development of arterial hypertension and to increased mortality [7-12].

Continuous positive airway pressure (CPAP) is the treatment of choice for patients with symptomatic OSAS, as it has been shown to improve daytime sleepiness, alertness and quality of life and to decrease blood pressure [13-15]. Whether CPAP treatment is effective in counteracting the autonomic imbalance and increased arterial stiffness in patients with OSAS remains a matter of debate.

The current authors have addressed this uncertainty by examining changes in 24-h urinary catecholamine excretion, BRS, arterial stiffness
AFFILIATIONS

*Oxford Centre for Respiratory Medicine, Churchill Hospital, - Dept of Cardiovascular Medicine, John Radcliffe Hospital, Oxford, and, \#Dept of Respiratory Medicine, Musgrove Park Hospital, Taunton, UK.

${ }^{+}$Both authors contributed equally to this study, and

${ }^{\S}$ Both authors contributed as senior authors.

\section{CORRESPONDENCE}

M. Kohler

Oxford Centre for Respiratory

Medicine

Oxford Radcliffe Hospitals

Churchill Hospital Campus Headington

Oxford

0X3 7LJ

UK

Fax: 441865225221

E-mail: Malcolm.K@bluewin.ch

Received:

February 202008

Accepted after revision:

July 092008

SUPPORT STATEMENT

Enrolment for this trial was finished before 2005.M. Kohler is a recipient of a European Respiratory Society research fellowship (No. 118) and a University of Zurich (Zurich, Switzerland) research fellowship.

STATEMENT OF INTEREST

A statement of interest for this study can be found at www.erj.ersjournals.com/misc/ statements.shtml 
and 24-h ambulatory blood pressure (ABP) in a double-blind, randomised controlled trial of therapeutic versus subtherapeutic CPAP in patients with symptomatic OSAS.

\section{METHODS}

\section{Patients}

Patients with possible obstructive sleep apnoea were referred to the Oxford Sleep Unit, (Oxford Centre for Respiratory Medicine, Oxford, UK) by general practitioners, ear, nose, and throat surgeons or other hospital consultants. Patients were eligible for the trial if they were CPAP-naïve males aged 2075 yrs with excessive daytime sleepiness (Epworth Sleepiness Score (ESS) $\geqslant 10$ ) [16] and proven obstructive sleep apnoea with $>10$ oxygen desaturations of $>4 \%$ per $h$ (oxygen desaturation index (ODI) $>10 \mathrm{~h}^{-1}$ ). All eligible patients were offered participation in the study, unless they required urgent CPAP therapy because of respiratory failure, driving or job issues. Data on blood pressure from 52 out of the 102 randomised patients had been used in a previously published study evaluating the effect of CPAP on ABP [15]. Measurements of urinary catecholamines, BRS and augmentation index were added to the previous protocol after receiving ethical approval and establishing the tests within the protocol. The study was approved by the Oxford research ethics committee (Oxford, UK; COREC No. 96.127) and written informed consent was obtained from all participants.

\section{Sleep study, CPAP and assessment of sleepiness}

OSAS was diagnosed from a 1-night in-hospital sleep study. Patients' body movements, cardiac frequency and pulse transit time (PTT) changes were recorded as measures of arousal from sleep. Pulse oximetry, snoring and increases in the respiratory swing in PTT were used as markers of breathing pattern and respiratory effort (Win-Visi monitoring system; Stowood Scientific Instruments, Oxford, UK) as previously described and validated $[15,17]$. The results of the sleep study were scored automatically, with manual review to ensure accuracy of the data. OSAS was diagnosed from review of all data and severity was quantified as the number of oxygen desaturations $>4 \%$ per $h$ study (ODI).

After enrolment, patients were randomly assigned to either therapeutic or subtherapeutic CPAP and then underwent a second sleep study, during which respiratory polygraphy was repeated and CPAP was used according to randomisation. In patients assigned to therapeutic CPAP, the therapeutic pressure was determined from overnight use of the Sullivan Autoset-T auto-adjusting CPAP machine (ResMed, Abingdon, UK), from which mask pressure was recorded and synchronised with the sleep study signals. The record was reviewed the following morning, and the optimum pressure to prevent sleep apnoea, usually the 95th percentile of pressure overnight, was determined by a sleep technician. Patients assigned to subtherapeutic CPAP used a machine that delivered $<1 \mathrm{cmH}_{2} \mathrm{O}$ pressure as previously described [15], which is insufficient to hold the pharynx open [13].

Patients remained blinded, whether they were receiving therapeutic or subtherapeutic CPAP, as did the investigators. The sleep nurses, who randomly assigned patients to the two groups, maintained the machines and assisted the patients, were not involved in outcome assessments.
Subjective sleepiness was assessed using the ESS, which assesses the tendency to fall asleep during eight typical daytime situations [16]. Objective sleepiness was measured with one sleep resistance challenge (the Oxford Sleep Resistance (Osler) test), which tests the subject's ability to stay awake in a darkened and sound-isolated room [18].

Patients were asked to refrain from caffeine and smoking on the day of examination. The sleep resistance challenge, pulse wave analysis and measurements of BRS were carried out on the same day and at the same time of day on the two occasions the patients were studied.

\section{Urine catecholamines}

Urine was collected for $24 \mathrm{~h}$ during normal daily activities at home. The adequacy of urine collections was evaluated by measures of volume and creatinine excretion. For analysis, $20 \mathrm{~mL}$ of urine were sampled and acidified with $0.5 \mathrm{~mL} \mathrm{50 \%}$ $\mathrm{HCl}$. Urine normetanephrine was measured by high-performance liquid chromatography as previously described [19]. Results were corrected for creatinine excretion $\left(\mu \mathrm{mol} \cdot \mathrm{mol}^{-1}\right.$ creatinine).

\section{BRS}

BRS was assessed using the bolus i.v. phenylephrine technique as previously described [20, 21]. Beat-by-beat arterial blood pressure was measured noninvasively by a finger cuff (Finapres, Ohmeda, CO, USA) [22]. For determination of the RR interval, a single-channel ECG was used (Hewlett-Packard 78342A; Hewlett-Packard, Palo Alto, CA, USA). Designated software (Foundation Salvatore Maugeri, Montescano, Italy) [21] was used to sample/digitise the finger blood pressure and ECG traces and detect systolic blood pressure and RR intervals, as well as for regression analysis. Phenylephrine (starting at $100 \mu \mathrm{g}$ per bolus, increasing by $25-\mu \mathrm{g}$ increments at 10 -min intervals to a maximum of $200 \mu \mathrm{g}$ per bolus) was administered i.v. with a minimum of three bolus injections to raise systolic arterial pressure by $15-40 \mathrm{mmHg}$.

\section{Pulse wave analysis}

The shape of the pressure waveform of an artery provides a measure of arterial stiffness and can be assessed by the technique of pulse wave analysis [23]. Radial artery pulse waveforms were recorded using a pressure tonometer and dedicated software as previously described (SphygmoCor; AtCor Medical, Sydney, Australia) [24]. Briefly, mean values of $\sim 10$ radial pulse waves are used to generate a corresponding central aortic pressure waveform with a validated mathematical transfer function [25]. The software uses an algorithm to determine the aortic pressure waveform's inflection point which corresponds to the onset of the reflected wave coming back from peripheral arteries, and divides the aortic pressure wave into an early and late systolic peak. Augmentation index, which quantifies augmentation of central aortic pressure (due to the reflected component of the pulse pressure waveform) and typically increases with age as the arteries become less compliant [26], is then calculated as the difference between the second $\left(P_{2}\right)$ and first systolic peak pressure $(P 1)$, expressed as percentage of the central pulse pressure $(P \mathrm{P})$ :

$$
\text { Augmentation index }(\%)=\left(\left(P_{2}-P_{1}\right) / P P\right) \times 100
$$


Essentially, the faster the pulse wave returned from the periphery, the stiffer the arteries, and the higher the calculated augmentation index.

A minimum of two sets of 10 radial artery pulse waveform readings were performed per patient until satisfactory operator indices, calculated by the software, were achieved, and the measurement with the highest operator indices was used for statistical analysis.

\section{Blood pressure}

For all patients, office blood pressure was measured in the sitting position with a standard digital automatic monitor (Omron Healthcare Company, Kyoto, Japan) at baseline and after 4 weeks of CPAP treatment. The mean value of three readings was used for analysis.

Validated recorders (TM 2420 and TM 2421; A\&D Engineering, Milpitas, CA, USA) were used to measure 24-h ABP during usual daily activities [27]. Monitors recorded blood pressure every $30 \mathrm{~min}$ throughout the $24 \mathrm{~h}$, and patients completed a diary card and pressed the event marker to identify the beginning of sleep and wake periods.

\section{Follow-up}

After baseline assessments, patients used their therapeutic or subtherapeutic CPAP machine (Sullivan 6; ResMed) for 4 weeks and then re-attended for repeat measurements of urine catecholamines, BRS, pulse wave analysis, blood pressure, ESS and the Osler test. Hour-meters on the CPAP machines were downloaded to calculate mean nightly use. At the end of the trial, CPAP pressure was retitrated in every patient to establish a therapeutic pressure for subsequent longterm use.

\section{Data analysis}

Data are expressed as mean $\pm \mathrm{SD}$, unless otherwise stated. Baseline characteristics of patients were compared between the therapeutic and subtherapeutic group using independent $t$ tests. Differences between and within groups, measured at baseline and after 4 weeks, were assessed with two-way repeated-measures ANOVA on an intention-to-treat basis, with no change assumed when follow-up data were missing. When data were analysed as per protocol, all statistically significant differences persisted, and therefore data from this analysis are not shown. For comparison of frequencies, the Chi-squared test of independence was used. Pearson's correlation analysis was used to evaluate the correlation between blood pressure, BRS, augmentation index and urine catecholamines. A p-value $<0.05$ was considered to be statistically significant.

\section{RESULTS}

\section{Trial profile and patients characteristics}

Figure 1 shows the trial profile. Overall, 102 patients with a mean age of $48.4 \pm 10.1$ yrs were randomised, 51 to therapeutic and 51 to subtherapeutic CPAP. The two groups were similar regarding age, body mass index, fat distribution, smoking status, prevalence of hypertension, proportion of patients on antihypertensive medication (and on $\beta$-blocking agents), diabetes and severity of OSAS, but the few subjects with coronary artery disease were only found in the therapeutic CPAP group (table 1).

\section{Urine catecholamines}

A total of 101 patients had their urine normetanephrine measured at baseline, and 96 at follow-up. Therapeutic CPAP reduced 24-h normetanephrine excretion significantly, compared with a nonsignificant increase in the subtherapeutic group. This reduction following therapeutic CPAP averaged $26 \%$ (table 2). Individual measurements of urine normetanephrine are shown in figure 2.

\section{BRS}

At baseline, 77 patients agreed to have their BRS measured, and 69 agreed at follow-up. In patients treated with therapeutic CPAP, BRS increased significantly compared with patients randomised to subtherapeutic CPAP. This increase following therapeutic CPAP averaged $24 \%$ (table 2; fig. 3). Just prior to actual BRS testing, heart rate and blood pressure were not significantly different between the therapeutic and subtherapeutic CPAP groups, both at baseline and follow-up.

\section{Pulse wave analysis}

At baseline, 72 patients agreed to have pulse wave analysis performed, and 62 agreed at follow-up. Mean change of augmentation index after 4 weeks of therapeutic CPAP was $-5.4 \pm 11.0 \%$, indicating a significant reduction in arterial stiffness, whereas augmentation index increased nonsignificantly by $+2.0 \pm 8.0 \%$ with subtherapeutic CPAP (table 2; fig. 4).

The change in augmentation index was not correlated with changes in 24-h urine normetanephrine excretion or change in BRS.

\section{Office and ambulatory blood pressure}

Office blood pressure was assessed in all 102 patients at baseline and in all 99 patients who finished the trial. A total of 62 patients agreed to have their 24-h ABP measured at baseline, and 53 at follow-up. Mean 24-h ABP fell by

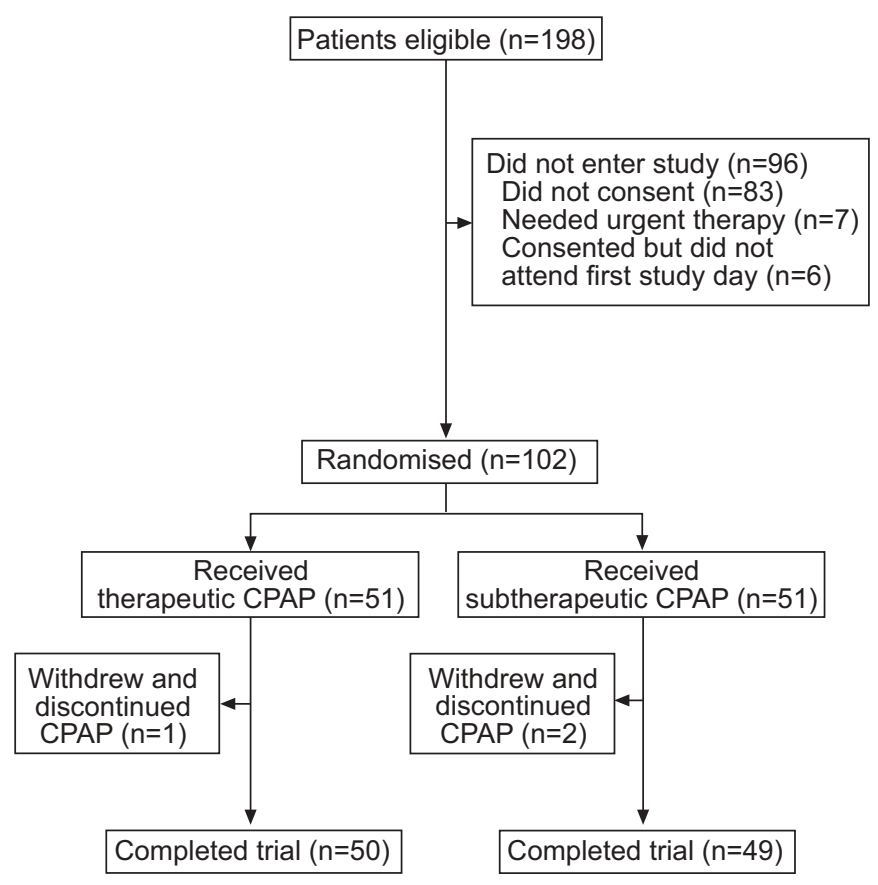

FIGURE 1. Trial profile. CPAP: continuous positive airway pressure. 
TABLE 1 Patient characteristics

\begin{tabular}{|c|c|c|c|}
\hline Patients n & 51 & 51 & \\
\hline Weight kg & $111.3 \pm 22.0$ & $115.5 \pm 25.1$ & 0.37 \\
\hline BMI $\mathbf{k g} \cdot \mathrm{m}^{-2}$ & $34.5 \pm 5.0$ & $35.8 \pm 7.3$ & 0.30 \\
\hline Neck circumference cm & $44.6 \pm 3.3$ & $45.1 \pm 4.0$ & 0.54 \\
\hline Ex-smokers \% & 54.9 & 43.2 & 0.24 \\
\hline Hypertensive \% & 25.5 & 21.6 & 0.64 \\
\hline Patients on antihypertensive therapy \% & 25.5 & 21.6 & 0.64 \\
\hline Diabetics \% & 2.0 & 2.0 & 1.00 \\
\hline Patients with coronary artery disease $\%$ & 0.0 & 7.4 & 0.04 \\
\hline Retitration CPAP pressure following study $\mathrm{cmH}_{2} \mathrm{O}$ & $10.1 \pm 1.6$ & $10.0 \pm 1.9$ & 0.72 \\
\hline
\end{tabular}

Data are presented as mean \pm SD, unless otherwise stated. CPAP: continuous positive airway pressure; BMI: body mass index; ESS: Epworth Sleepiness Score; Osler Oxford sleep resistance.

$2.6 \mathrm{mmHg}$ with therapeutic CPAP, whereas no significant change was found with subtherapeutic CPAP. Details of blood pressure measurements are given in table 2.
In the therapeutic CPAP group, no correlation was found between changes in mean 24-h blood pressure and those in BRS, urinary normetanephrines or augmentation index.

TABLE 2 Blood pressure (BP), baroreflex sensitivity (BRS), urine normetanephrine and augmentation index before and after treatment

\begin{tabular}{|c|c|c|c|c|c|}
\hline & \multicolumn{2}{|c|}{ Subtherapeutic CPAP } & \multicolumn{2}{|c|}{ Therapeutic CPAP } & \multirow[t]{2}{*}{ p-value ${ }^{\#}$} \\
\hline & Baseline & Follow-up & Baseline & Follow-up & \\
\hline Office mean BP mmHg & $109.3 \pm 11.7$ & $107.3 \pm 11.2$ & $104.2 \pm 12.6$ & $102.4 \pm 8.8$ & 0.81 \\
\hline Office systolic BP mmHg & $141.6 \pm 17.4$ & $140.4 \pm 17.4$ & $135.8 \pm 16.0$ & $133.0 \pm 12.6$ & 0.46 \\
\hline Office diastolic BP $\mathrm{mmHg}$ & $92.9 \pm 11.4$ & $90.8 \pm 10.2$ & $89.3 \pm 10.8$ & $87.0 \pm 8.9$ & 0.96 \\
\hline 24-h diastolic BP" $\mathrm{mmHg}$ & $88.3 \pm 8.1$ & $88.9 \pm 8.0$ & $83.9 \pm 9.3$ & $81.3 \pm 9.8$ & 0.04 \\
\hline Wake mean BP mmHg & $107.9 \pm 10.9$ & $110.0 \pm 10.2$ & $103.2 \pm 9.2$ & $100.1 \pm 10.2$ & 0.002 \\
\hline Wake systolic BP $\mathrm{mmHg}$ & $142.6 \pm 21.5$ & $145.4 \pm 20.0$ & $135.9 \pm 14.0$ & $133.1 \pm 14.6$ & 0.02 \\
\hline Wake diastolic BP mmHg & $90.7 \pm 7.9$ & $92.3 \pm 7.5$ & $86.9 \pm 8.9$ & $83.8 \pm 10.1$ & 0.005 \\
\hline Sleep mean BP mmHg & $99.3 \pm 11.8$ & $98.5 \pm 9.9$ & $92.0 \pm 13.1$ & $89.3 \pm 11.0$ & 0.35 \\
\hline Sleep systolic BP mmHg & $131.1 \pm 21.3$ & $129.8 \pm 15.9$ & $121.5 \pm 17.0$ & $117.4 \pm 16.8$ & 0.33 \\
\hline Augmentation ${ }^{f}$ index \% & $12.2 \pm 13.6$ & $14.2 \pm 14.9$ & $14.5 \pm 11.3$ & $9.1 \pm 13.8$ & 0.001 \\
\hline
\end{tabular}

Data are presented as mean $\pm S D$, unless otherwise stated. For both groups $n=51$. CPAP: continuous positive airway pressure. \#: calculated by two-way ANOVA for repeated measurements and compares treatment effects; ": 24-h BP was measured in 33 patients in the therapeutic, and in 29 in the subtherapeutic group; ${ }^{+}$: baroreflex sensitivity was measured in 34 patients in the therapeutic, and in 43 in the subtherapeutic group; ${ }^{\text {s. }}$ : urine normetanephrine was measured in 51 patients in the therapeutic, and in 50 patients in the subtherapeutic group; ${ }^{f}$ : augmentation index was measured in 30 patients in the therapeutic, and 42 patients in the subtherapeutic group. 

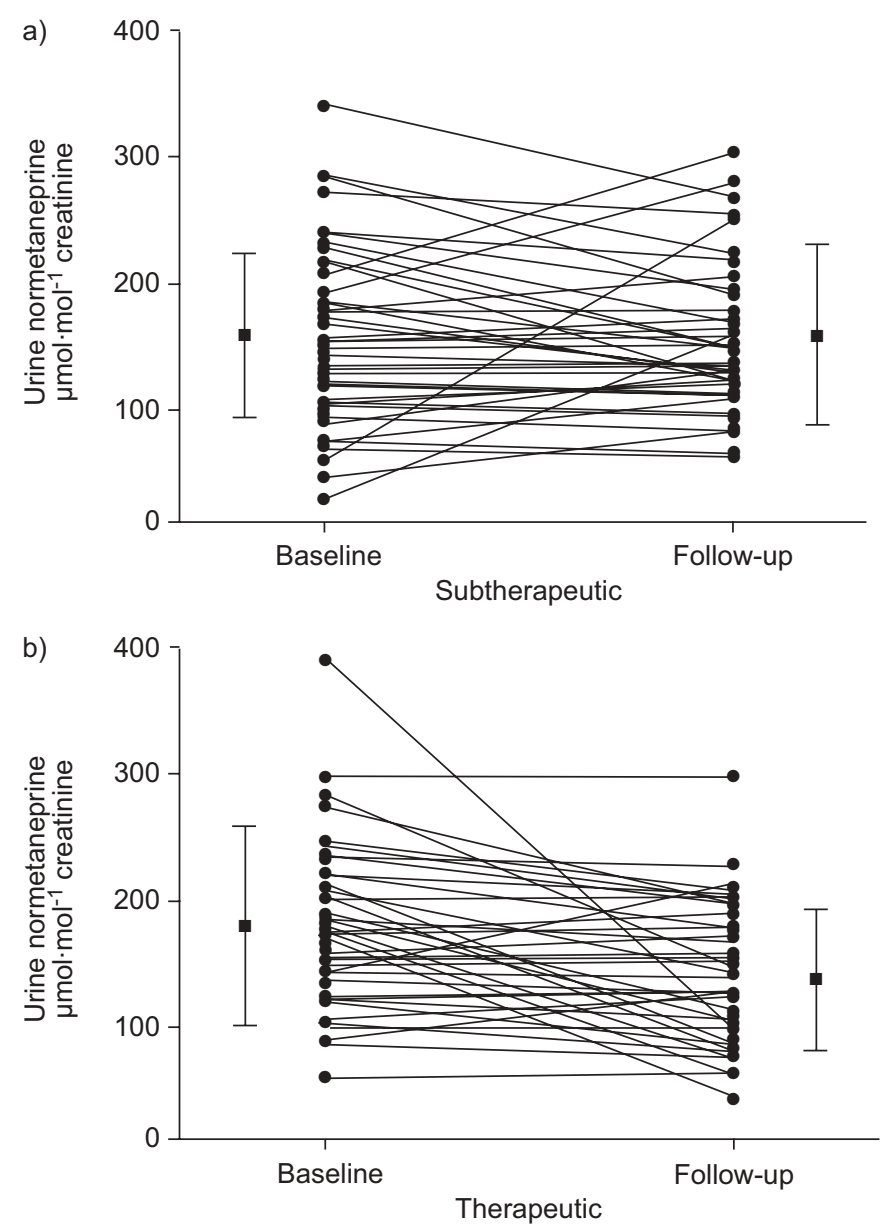

FIGURE 2. Individual urine normetanephrine levels at baseline and follow-up in a) the subtherapeutic continuous positive airway pressure (CPAP) group and b) the therapeutic CPAP group. Two-way ANOVA for repeated measurements revealed that therapeutic CPAP significantly reduced normetanephrine excretion compared with subtherapeutic CPAP $(p=0.004)$. $\mathbf{~ : ~ m e a n ; ~ v e r t i c a l ~ b a r s : ~ S D . ~}$

\section{Measures of sleepiness and CPAP compliance}

Therapeutic CPAP significantly reduced the ESS from $15.8 \pm 4.0$ to $6.8 \pm 5.1$ (difference $-8.6,95 \%$ confidence interval (CI) -10.2- -7.1; p <0.0001) and improved objective sleepiness measured by the Osler test from $18.1 \pm 13.1$ to $26.8 \pm 12.9 \mathrm{~min}$ (difference $8.7 \mathrm{~min}, 95 \%$ CI $4.5-12.9 ; \mathrm{p}=0.009$ ). Subjective sleepiness measured by ESS also improved with subtherapeutic CPAP, from $15.2 \pm 4.0$ to $11.9 \pm 5.9$ (difference $-3.3,95 \% \mathrm{CI}$ $-4.6--2.0 ; \mathrm{p}<0.0001)$. However, subtherapeutic CPAP had no significant effect on objective sleepiness $(17.3 \pm 13.1$ and $18.3 \pm 14.3 \mathrm{~min}$; difference $1.0 \mathrm{~min}, 95 \% \mathrm{CI}-2.7-4.7 ; \mathrm{p}=0.58$ ). Compliance with CPAP did not differ between the two groups (table 1). The present data show a clear difference in the change of objective sleepiness between the two groups, despite a placebo effect on ESS.

In the therapeutic CPAP group, the improvement of objective sleepiness assessed by the Osler test was correlated with 24-h diastolic blood pressure fall $(\mathrm{r}=-0.48,95 \%$ CI $-0.72--0.14$; $\mathrm{n}=29 ; \mathrm{p}=0.008$ ), but not with change in BRS, urine normetanephrine excretion or augmentation index.
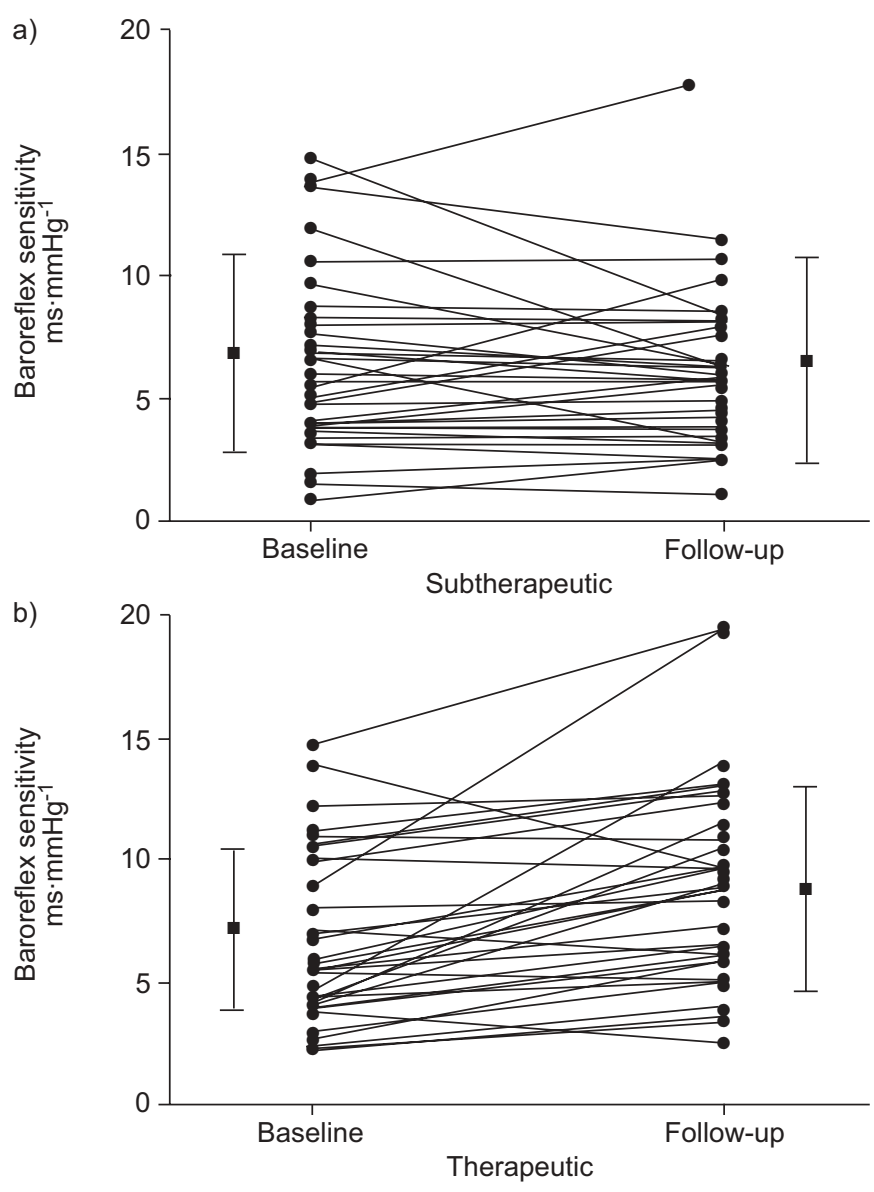

FIGURE 3. Individual baroreflex sensitivity at baseline and after 4 weeks of a) subtherapeutic continuous positive airway pressure (CPAP) and b) therapeutic CPAP. ANOVA showed that therapeutic CPAP significantly increased baroreflex sensitivity compared with subtherapeutic CPAP ( $p=0.001)$. $\mathbf{q}$ : mean; vertical bars: SD.

\section{DISCUSSION}

The present authors have performed a large randomised controlled trial on the effects of CPAP on daytime BRS and arterial stiffness in patients with OSAS, and found a significant improvement in these parameters after 4 weeks of active treatment. Furthermore, therapeutic CPAP treatment decreased 24-h urine normetanephrine excretion, consistent with a reduction in sympathetic nerve activity, and mean 24-h ABP. These beneficial changes may explain the increased survival found in OSAS patients treated with CPAP in a recently published observational study [12].

\section{Sympathetic activity}

Elevated levels of circulating plasma norepinephrine and urine catecholamine metabolites have been demonstrated during sleep and resting waking periods in patients with OSAS [28, 29]. It has been suggested that hypoxia and frequent periodic arousals from sleep may underlie the increased sympathetic nervous activity in the presence of OSAS, although arousals seem to be more significant $[10,30]$. Consistent with a reduction of sympathetic nerve activity, the present authors found that 4 weeks of CPAP 

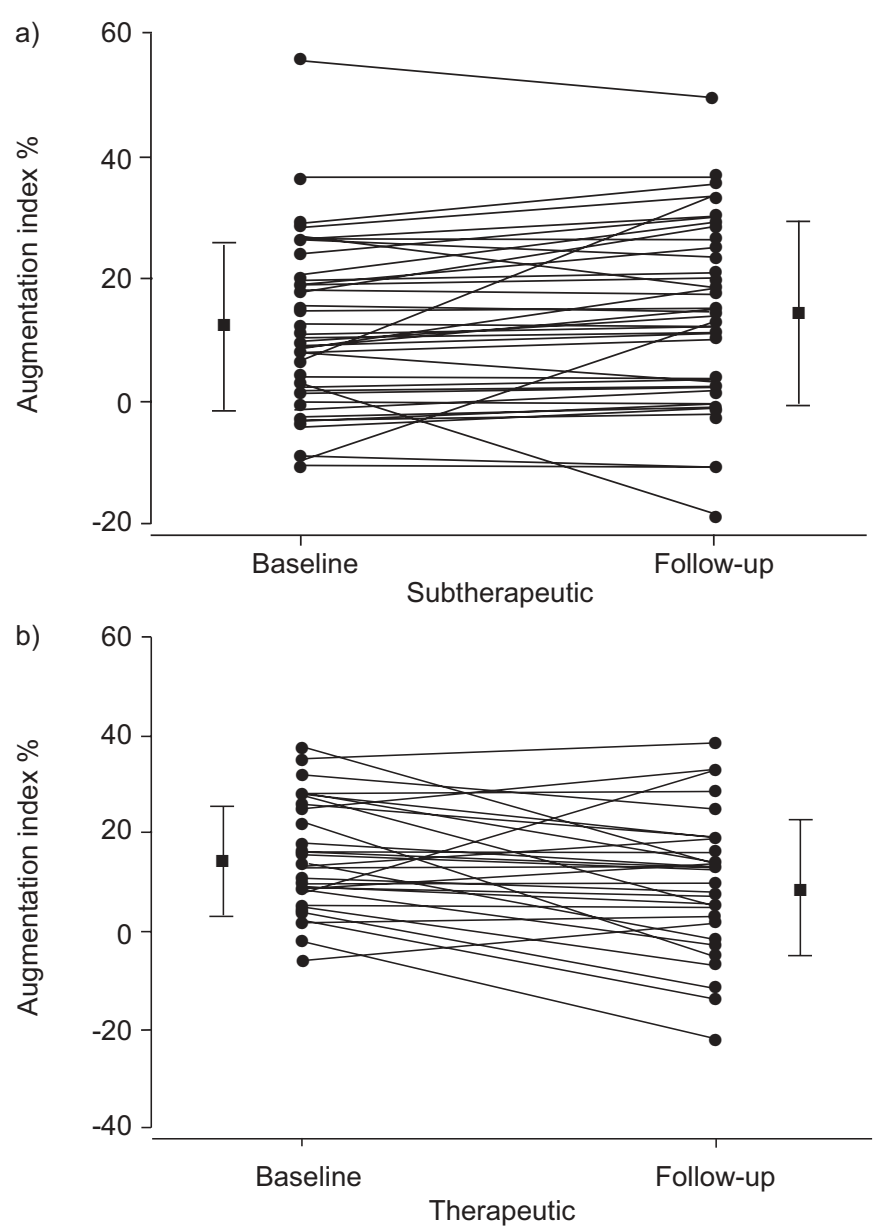

FIGURE 4. Individual augmentation index assessed by pulse wave analysis at baseline and follow-up in a) the subtherapeutic continuous positive airway pressure (CPAP) group and b) the therapeutic CPAP group. ANOVA showed that therapeutic CPAP significantly decreased augmentation index compared with subtherapeutic $\operatorname{CPAP}(p=0.001)$. $\mathbf{~ : ~ m e a n ; ~ v e r t i c a l ~ b a r s : ~ S D . ~}$

therapy decreased 24-h urine normetanephrine excretion by $26 \%$. This finding is supported by results from an uncontrolled study from HEDNER et al. [31], who found significantly decreased levels (32-54\%) of urine vanylmandelic acid and metanephrines after 20.5 (range 14-26) months of CPAP in patients with severe OSAS. Furthermore, in agreement with the current study, ZIEGLER et al. [32] found a $26 \%$ reduction in 24-h urine norepinephrine after 7 days of CPAP in a randomised controlled trial including 38 patients with moderate-to-severe OSAS. The observed effect of $\mathrm{CPAP}$ on urinary catecholamines is comparable to that achieved by weight-reducing gastroplasty (reduction of the body mass index from 38.6 to $28.5 \mathrm{~kg} \cdot \mathrm{m}^{-2}$ produces a $23 \%$ decrease in norepinephrine excretion) [33] or by endurance training programmes of $>4$ weeks' duration (reduction of plasma norepinephrine 29\%) [34]. In addition to potential beneficial effects on heart rhythm disturbances, which have been associated with enhanced sympathetic activity, CPAP might also have a direct effect on vascular properties, since the level of sympathetic nerve activity has been shown, for example, to be associated with femoral artery wall thickness in healthy humans [35].

\section{BRS}

Reduced vagal activity and relative sympathetic predominance have been shown to be associated with sudden death and poor prognosis in patients after myocardial infarction. [21, 36] BRS, a well-established index of cardiac vagal responsiveness [37], has been shown to be depressed during sleep in patients with severe OSAS, and uncontrolled short-term trials have suggested an improvement of nocturnal BRS with CPAP treatment [7-9]. In the present study, BRS measured during daytime increased by $24 \%$ after 4 weeks of therapeutic CPAP, indicating that the beneficial effect of treatment on the cardiac sympatho-vagal balance is not limited to the sleep period. It should be noted that this improvement in BRS after CPAP is comparable in size to the effect seen after several months of endurance training in patients with mild hypertension [38]. The increase of BRS in response to CPAP might contribute to the improved survival found in OSAS patients treated with CPAP, compared with untreated patients [12], as decreased vagal tone and increased sympathetic activity may lead to arrhythmias and a peak in sudden death from cardiac causes during the sleeping hours in patients with OSAS [39].

\section{Arterial stiffness}

Augmentation index, a measure of central arterial stiffness and pressure wave reflection, independently predicts cardiovascular events in high-risk populations [40]. In the current study, augmentation index decreased significantly from 14.5 to $9.1 \%$ after 4 weeks of CPAP therapy. This considerable reduction is comparable in size to the effect seen after 12 weeks of exercise training in patients with coronary artery disease [41] or after 6 weeks of eprosartan (600 mg daily) in patients with nevertreated arterial hypertension [42]. The mechanism responsible for this fall in augmentation index is at present a subject of speculation. A decrease in sympathetic activity may contribute to the observed reduction in arterial stiffness; however, the change in urine normetanephrine excretion with CPAP did not correlate with the change in augmentation index in the present study. Alternatively, augmentation index has been shown to increase with the plasma level of asymmetric dimethylarginine (an endogenous inhibitor of endothelial nitric oxide synthase), suggesting that improved endothelial function secondary to a higher bioavailability of nitric oxide may be a further beneficial effect of CPAP [43].

\section{Blood pressure measurements}

The current results on 24-h ABP showed a significant fall of $2.6 \mathrm{mmHg}$ in mean blood pressure after 4 weeks of therapeutic $\mathrm{CPAP}$, in agreement with other randomised controlled trials [44]. It is important to point out that the data presented on 24-h $\mathrm{ABP}$ has been included in a previously published study evaluating the effect of CPAP on ABP [15], and therefore the current report does not provide new cases for meta-analyses.

The reductions were similar for systolic and diastolic ABP during wake times, but the reductions seen during sleep failed to achieve statistical significance. This discrepancy may be explained by the observation that nocturnal blood pressure measurements with automatic inflation devices produce electroencephalic evidence of arousals from sleep, and probably underestimate the true reduction in blood pressure during sleep [45]. 
Interestingly, therapeutic CPAP did not significantly lower office blood pressure when compared to subtherapeutic CPAP in current study, a finding similar to that of MONASTERIO et al. [46], who did not observe a reduction in office blood pressure after 6 weeks of CPAP in patients with mild OSAS. These findings are likely to be due to difficulties in detecting small changes in blood pressure with the less reproducible office measurements [47], often ascribed to variable "white coat" effects [47]. The significant correlation found in the present study between the improvement in sleepiness and the reduction in blood pressure supports a possible consequence of recurrent arousals from sleep.

\section{Study limitations}

There are some limitations of the current study which have to be mentioned. According to common clinical practice [48], a single titration night was used to determine the required level of CPAP to abolish OSAS, and the sleep study was not repeated at the end of the trial to ascertain that patients were optimally treated. Therefore, the study design did not allow the impact of therapeutic CPAP on arousal frequency to be quantified, which might have enabled an exploration of the possibility that arousal frequency is a major determinant of sympathetic activity in patients with OSA. However, the large improvement in objective sleepiness in the therapeutic CPAP group strongly suggests that patients in the active CPAP group were adequately treated.

The current study used an ODI $>10 \mathrm{~h}^{-1}$ associated with excessive daytime sleepiness (ESS $>10$ ) instead of the more commonly used apnoea/hypopnoea index (AHI) to define OSAS. However, there is good evidence that equivalence exists between the two measures of OSAS severity [49], and indeed ODI is at least as reproducible as the AHI between repeated nights [50]. Furthermore, oscillations in oxygen levels may be more pertinent to the vascular effects of OSA than apnoeic events [51] and therefore the current authors believe that the use of an ODI rather than an AHI does not limit the interpretation of their data.

Only males with moderate-to-severe OSAS were included in the present study, and the findings might therefore not be applicable to females, or patients with milder forms of OSAS. Therefore, further randomised controlled studies are needed to prove that CPAP does produce the same benefits on measures of cardiovascular risk in females, or patients with mild OSAS.

\section{CONCLUSIONS}

In a randomised controlled trial of therapeutic versus subtherapeutic continuous positive airway pressure in male patients with moderate to severe obstructive sleep apnoea syndrome, the present authors have shown that active treatment is associated with a significant decrease in 24-h urinary catecholamine excretion, arterial stiffness and ambulatory blood pressure, as well as with an improvement in baroreflex sensitivity. These findings suggest that treatment of symptomatic obstructive sleep apnoea syndrome patients with continuous positive airway pressure may have a positive impact on patients' survival by effectively reducing a number of well-established risk factors for cardiovascular disease.

\section{REFERENCES}

1 Young T, Peppard PE, Gottlieb DJ. Epidemiology of obstructive sleep apnea: a population health perspective. Am J Respir Crit Care Med 2002; 165: 1217-1239.

2 Peker Y, Hedner J, Norum J, Kraiczi H, Carlson J. Increased incidence of cardiovascular disease in middle-aged men with obstructive sleep apnea: a 7-year follow-up. Am J Respir Crit Care Med 2002; 166: 159-165.

3 Zwillich C, Sinoway L. Surges of muscle sympathetic nerve activity during obstructive apnea are linked to hypoxemia. J Appl Physiol 1995; 79: 581-588.

4 Somers VK, Kyken ME, Clary MP, Abbound FM. Sympathetic neural mechanisms in obstructive sleep apnea. J Clin Invest 1995; 96: 1897-1904.

5 Jelic S, Bartels MN, Mateika JH, Ngai P, DeMeersman RE, Basner RC. Arterial stiffness increases during obstructive sleep apneas. Sleep 2002; 25: 15-20.

6 Lovett JK, Rothwell PM. Site of carotid plaque ulceration in relation to direction of blood flow: an angiographic and pathological study. Cerebrovasc Dis 2003; 16: 369-375.

7 Bonsignore MR, Parati G, Insalaco G, et al. Continuous positive airway pressure treatment improves baroreflex control of heart rate during sleep in severe obstructive sleep apnea syndrome. Am J Respir Crit Care Med 2002; 166: 279-286.

8 Ryan S, Ward S, Heneghan C, McNicholas WT. Predictors of decreased spontaneous baroreflex sensitivity in obstructive sleep apnea syndrome. Chest 2007; 131: 1100-1107.

9 Bonsignore MR, Parati G, Insalaco G, et al. Baroreflex control of heart rate during sleep in severe obstructive sleep apnoea: effects of acute CPAP. Eur Respir J 2006; 27: 128-135.

10 Carlson JT, Hedner J, Elam M, Ejnell H, Sellgren J, Wallin BG. Augmented resting sympathetic activity in awake patients with obstructive sleep apnea. Chest 1993; 103: 1763-1768.

11 Elmasry AE, Lindberg E, Hedner J, Janson C, Boman G. Obstructive sleep apnoea and urine catecholamines in hypertensive males: a population-based study. Eur Respir J 2002; 19: 511-517.

12 Marin JM, Carrizo SJ, Vicente E, Agusti AG. Long-term cardiovascular outcomes in men with obstructive sleep apnoea-hypopnoea with or without treatment with continuous positive airway pressure: an observational study. Lancet 2005; 365: 1046-1053.

13 Jenkinson C, Davies RJ, Mullins R, Stradling JR. Comparison of therapeutic and subtherapeutic nasal continuous positive airway pressure for obstructive sleep apnoea: a randomised prospective parallel trial. Lancet 1999; 353: 2100-2105.

14 Hack M, Davies RJ, Mullins R, et al. Randomised prospective parallel trial of therapeutic versus subtherapeutic nasal continuous positive airway pressure on simulated steering performance in patients with obstructive sleep apnoea. Thorax 2000; 55: 224-231.

15 Pepperell JCT, Ramdassingh-Dow S, Crosthwaite N, et al. Ambulatory blood pressure after therapeutic and subtherapeutic nasal continuous positive airway pressure for obstructive sleep apnoea: a randomised parallel trial. Lancet 2002; 359: 204-210. 
16 Johns MW. A new method for measuring daytime sleepiness: the Epworth sleepiness scale. Sleep 1991; 14: 540-545.

17 Pitson DJ, Stradling JR. Autonomic markers of arousal during sleep in patients undergoing investigation for obstructive sleep apnoea, their relationship to EEG arousals, respiratory events and subjective sleepiness. J Sleep Res 1998; 7: 53-60.

18 Bennett LS, Stradling JR, Davies RJ. A behavioural test to assess daytime sleepiness in obstructive sleep apnoea. J Sleep Res 1997; 6: 142-145.

19 Peaston RT. Routine determination of urinary catecholamines by high-performance liquid chromatography with electrochemical detection. J Chromatogr 1988; 424: 263-272.

20 La Rovere MT, Specchia G, Mortara A, Schwartz PJ. Baroreflex sensitivity, clinical correlates and cardiovascular mortality among patients with a first myocardial infarction: a prospective study. Circulation 1988; 78: 816-824.

21 La Rovere MT, Bigger JT, Marcus FI, Mortara A, Schwartz PJ. Baroreflex sensitivity and heart-rate variability in prediction of total cardiac mortality after myocardial infarction. ATRAMI (Autonomic Tone and Reflexes After Myocardial Infarction) Investigators. Lancet 1998; 351: 478-484.

22 Parati G, Casadei R, Groppelli A, Di Rienzo M, Mancia G. Comparison of finger and intra-arterial blood pressure monitoring at rest and during laboratory testing. Hypertension 1989; 13: 647-655.

23 O'Rourke MF, Gallagher DE. Pulse wave analysis. J Hypertens 1996; 14: 147-157.

24 Kelly RP, Hayward CS, Avolio AP, O'Rourke M. Noninvasive determination of age-related changes in the human arterial pulse. Circulation 1989; 80: 1652-1659.

25 Pauca AL, O'Rourke MF, Kon ND. Prospective evaluation of a method for estimating ascending aortic pressure from the radial artery pressure waveform. Hypertension 2001; 38: 932-937.

26 Safar ME, London GM. Therapeutic studies and arterial stiffness in hypertension: recommendations of the European Society of Hypertension. The clinical committee of arterial structure and function. Working group on vascular structure and function of the European Society of Hypertension. J Hypertens 2000; 18: 1527-1535.

27 O'Brien E, Mee F, Atkins N, O'Malley K. Accuracy of the Takeda TM-2420/TM-2020 determined by the British Hypertension Society protocol. J Hypertens 1991; 9: 571-572.

28 Fletcher EC, Miller J, Schaaf JW, Fletcher JG. Urinary catecholamines before and after tracheostomy in patients with obstructive sleep apnea and hypertension. Sleep 1987; 10: $35-44$.

29 Eisenberg E, Zimlichman R, Lavie P. Plasma norepinephrine levels in patients with sleep apnea syndrome. $N$ Engl J Med 1990; 322: 932-933.

30 Norman D, Loredo JS, Nelesen RA, et al. Effects of continuous positive airway pressure versus supplemental oxygen on 24-hour ambulatory blood pressure. Hypertension 2006; 47: 840-845.

31 Hedner J, Darpo B, Ejnell H, Carlson J, Caidahl K. Reduction in sympathetic acitivity after long-term CPAP treatment in sleep apnoea: cardiovascular implications. Eur Respir J 1995; 8: 222-229.
32 Ziegler MG, Mills PJ, Loredo JS, Ancoli-Israel S, Dimsdale JE. Effect of continuous positive airway pressure and placebo treatment on sympathetic nervous activity in patients with obstructive sleep apnea. Chest 2001; 120: 887-893.

33 Karason K, Molgaard H, Wikstrand J, Sjostrom L. Heart rate variability in obesity and the effect of weight loss. Am J Cardiol 1999; 83: 1242-1247.

34 Cornelissen VA, Fagard RH. Effects of endurance training on blood pressure, blood pressure-regulating mechanisms, and cardiovascular risk factors. Hypertension 2005; 46: 667-675.

35 Dinenno FA, Jones PP, Seals DR, Tanaka H. Age-associated arterial wall thickening is related to elevations in sympathetic activity in healthy humans. Am J Physiol Heart Circ Physiol 2000; 278: H1205-1210.

36 Volpi A, De Vita C, Franzosi MG, et al. Determinants of 6month mortality in survivors of myocardial infarction after thrombolysis: results of the GISSI-2 data base. Circulation 1993; 88: 416-429.

37 La Rovere MT, Mortara A, Schwartz PJ. Baroreflex sensitivity. J Cardiovasc Electrophysiol 1995; 6: 761-774.

38 Somers VK, Conway J, Johnston J, Sleight P. Effects of endurance training on baroreflex sensitivity and blood pressure in borderline hypertension. Lancet 1991; 337: 1362-1368.

39 Gami AS, Howard DE, Olson EJ, Somers VK. Day-night pattern of sudden death in obstructive sleep apnea. $N$ Engl J Med 2005; 352: 1206-1214.

40 Weber T, Auer J, O'Rourke MF, et al. Arterial stiffness, wave reflections, and the risk of coronary artery disease. Circulation 2004; 109: 184-189.

41 Edwards DG, Schofield RS, Magyari PM, Nichols WW, Braith RW. Effect of exercise training on central aortic pressure wave reflection in coronary artery disease. Am J Hypertens 2004; 17: 540-543.

42 Dhakam Z, McEniery CM, Yasmin, Cockroft JR, Brown MJ, Wilkinson IB. Atenolol and eprosartan; differential effects on central blood pressure and aortic pulse wave velocity. Am J Hypertens 2006; 19: 214-219.

43 Ip MSM, Tse HF, Lam B, Tsang KWT, Lam WK. Endothelial function in obstructive sleep apnea and response to treatment. Am J Respir Crit Care Med 2004; 169: 348-353.

44 Faccenda JF, Mackay TW, Boon NA, Douglas NJ. Randomized placebo-controlled trial of continuous positive airway pressure on blood pressure in the sleep apneahypopnea syndrome. Am J Respir Crit Care Med 2001; 163: 344-348.

45 Davies RJO, Jenkins NE, Stradling JR. Effect of measuring ambulatory blood pressure on sleep and on blood pressure during sleep. BMJ 1994; 308: 820-823.

46 Monasterio C, Vidal S, Duran J, et al. Effectiveness of continuous positive airway pressure in mild sleep apneahypopnea syndrome. Am J Respir Crit Care Med 2001; 164: 939-943.

47 Parati G, Valentini M. Do we need out-of-office blood pressure in every patient? Curr Opin Cardiol 2007; 22: 321-328.

48 Masa JF, Jimenez A, Duran J, et al. Alternative methods of titrating continuous positive airway pressure. Am J Respir Crit Care Med 2004; 170: 1218-1224. 
49 Vazquez JC, Tsai WH, Flemons WW, et al. Automated analysis of digital oximetry in the diagnosis of obstructive sleep apnoea. Thorax 2000; 55: 302-307.

50 Stradling J, Smith D, Radulovacki M, Carley D. Effect of ondansetron on moderate obstructive sleep apnea, a single night, placebo-controlled trial. J Sleep Res 2003; 12: 169-170.

51 Dematteis M, Julien C, Guillermet C, et al. Intermittent hypoxia induces early functional cardiovascular remodeling in mice. Am J Respir Crit Care Med 2008; 177: 227-235. 